\title{
HE 1327-2326, AN UNEVOLVED STAR WITH [Fe/H] < -5.0. III. DOES ITS ATMOSPHERE REFLECT ITS NATAL COMPOSITION?
}

\author{
A. J. Korn ${ }^{1}$, O. Richard ${ }^{2}$, L. Mashonkina ${ }^{3,4}$, M. S. Bessell ${ }^{5}$, A. Frebel ${ }^{6,7}$, And W. Aoki ${ }^{8}$ \\ ${ }^{1}$ Department of Physics and Astronomy, Division of Astronomy and Space Physics, Uppsala University, Box 515, 75120 Uppsala, Sweden; \\ andreas.korn@fysast.uu.se \\ 2 GRAAL, Université Montpellier II, CNRS, Place E. Bataillon, 34095 Montpellier, France \\ ${ }^{3}$ Institute of Astronomy, Russian Academy of Science, Pyatnitskaya 48, 119017 Moscow, Russia \\ ${ }^{4}$ Institut für Astronomie und Astrophysik der Universität München, Scheinerstr. 1, 81679 München, Germany \\ ${ }^{5}$ RSAA, The Australian National University, Mt. Stromlo, Cotter Rd., Weston, ACT 2611, Australia \\ ${ }^{6}$ McDonald Observatory, University of Texas, 1 University Station, C1400, Austin, TX 78712-0259, USA \\ ${ }^{7}$ Harvard-Smithsonian Center for Astrophysics, 60 Garden St., MS-20, Cambridge, MA 02138, USA \\ ${ }^{8}$ National Astronomical Observatory of Japan, Mitaka, Tokyo 181-8588, Japan \\ Received 2008 December 19; accepted 2009 March 26; published 2009 May 20
}

\begin{abstract}
Based on spectroscopic constraints derived from nonlocal thermodynamic equilibrium line formation, we explore the likely range of stellar parameters $\left(T_{\text {eff }}\right.$ and $\log g$ ) for the hyper-metal-poor (HMP) star HE $1327-2326$. Combining the constraints from Balmer line profiles and the CaI/II ionization equilibrium, a subgiant stage of evolution is indicated. This result is further supported by spectrophotometric observations of the Balmer jump. If a higher $T_{\text {eff }}$ value was used (as favored by some photometric calibrations), the spectroscopic analysis would indicate a turnoff-point stage of evolution. Using a stellar-structure code that treats the effects of atomic diffusion throughout the star in detail, we evolve a low-mass model star to reach the HertzsprungRussell-diagram position of HE 1327-2326 after roughly 13 Gyr. While the surface abundances are modified significantly (by more than 1 dex for the case of uninhibited diffusion), such corrections cannot resolve the discrepancy between the abundance inferred from the nondetection of the Li I resonance line at $6707 \AA$ and the Wilkinson Microwave Anisotropy Probe based primordial lithium abundance. As there are numerous processes that can destroy lithium, any cosmological interpretation of a lower-than-expected lithium abundance at the lowest metallicities will have to await sample sizes of unevolved HMP stars that are 1 order of magnitude larger. The situation remains equally inconclusive concerning atomic-diffusion corrections. Here, attempts have to be made to better constrain internal mixing processes, both observationally and by means of sophisticated modeling. With constraints on additional mixing processes taken from a recent globular-cluster study, the likeliest scenario is that HE 1327-2326's surface abundances have undergone mild depletion (of order 0.2 dex).
\end{abstract}

Key words: diffusion - stars: abundances - stars: evolution - stars: fundamental parameters - stars: individual (HE 1327-2326) - stars: Population II

Online-only material: color figure

\section{INTRODUCTION}

The most metal-poor stars provide us with chemical information about the early universe and the onset of the cosmic chemical evolution. This is based on the assumption that the surface abundances of these old stars indeed reflect the composition of their birth cloud, and have not changed during the long lifetimes. In principle, there are several internal and external processes which may have an impact on the surface composition of old stars, such as internal pollution of the outer layers by nuclear burning products from the stellar interior (in case the star is sufficiently evolved) or accretion of interstellar gas during $\sim 13$ Gyr of orbiting though the Galactic potential (the latter process was recently shown to be rather inefficient; see Frebel et al. 2008b).

HE $1327-2326$ is a low-mass star with extreme properties: its excessively low metallicity in terms of iron $([\mathrm{Fe} / \mathrm{H}] \approx$ -5.6; Frebel et al. 2005) is accompanied by large (3-4 dex) overabundances of the elements $\mathrm{C}, \mathrm{N}$, and $\mathrm{O}$ relative to iron, a property shared by the other two stars known in this metallicity regime (Christlieb et al. 2002; Norris et al. 2007). What these stars can tell us about the properties of the first generation of stars (metal-free Population III) is still debated. The abundance pattern has been interpreted to reflect the nucleosynthetic yields of a single Pop. III supernova (Iwamoto et al. 2005; Meynet et al. 2006), but see Venn \& Lambert (2008) for an alternative scenario potentially explaining the abundance peculiarities of hyper-metal-poor (HMP) stars.

Apart from these interpretative uncertainties, our ability to read the chemical fingerprints encoded in the spectra of these stars is limited by the technical challenge to properly model the line-formation process taking place in their atmospheres. For the most part, the chemical abundances have been inferred using one-dimensional (1D) hydrostatic model atmospheres in local thermodynamic equilibrium (LTE) with rough (estimated) corrections for departures from LTE (so-called NLTE effects). In the case of calcium, the only element observed in two ionization stages, 1D LTE leads to a clear mismatch for the abundances derived from $\mathrm{Ca}$ I and $\mathrm{Ca}$ II: the abundance from the $\mathrm{Ca}$ I resonance line at $4226 \AA$ gives an abundance 0.57 dex below the abundance derived from the Ca II K line at $3934 \AA$ (Aoki et al. 2006). This may reflect the shortcomings of classical 1D LTE analyses when applied to HMP stars. Explorations using 3D hydrodynamic model atmospheres have modified the abundance ratios (bringing down the large overabundances by typically 0.7 dex for the $\mathrm{CNO}$ elements), but have been unable to resolve 
the above-mentioned discrepancy (reported by Frebel et al. (2008a) to be 0.46 dex in 1D LTE and 0.47 dex in 3D LTE).

The LTE assumption is particularly questionable when analyzing (hyper-)metal-poor stars, as their atmospheres are ultraviolet (UV) transparent leading to strong photoionization. Furthermore, electronic collisions are less efficient due to the reduced number of electron donors like $\mathrm{Fe}$ and $\mathrm{Mg}$. Both these effects will drive the statistical equilibrium away from LTE. It is thus worthwhile to check whether NLTE can solve the obvious mismatch for the case of calcium.

HE 1327-2326 is either an evolved main-sequence (MS) or early subgiant (SG) star at $T_{\text {eff }} \approx 6200 \mathrm{~K}$, i.e., HE $1327-2326$ is a star on the Spite plateau of lithium (Spite \& Spite 1982) formed by stars with $T_{\text {eff }} \geqslant 5800 \mathrm{~K}$ and $[\mathrm{Fe} / \mathrm{H}] \leqslant-1.3$ (Charbonnel $\&$ Primas 2005). Despite the uncertainty in the evolutionary status of HE 1327-2326, the relatively unevolved nature of this old star makes it an ideal target for a $\mathrm{Li}$ measurement. Other unevolved metal-poor stars have been used to infer the primordial $\mathrm{Li}$ abundance. However, the Li doublet at $6707 \AA$ could not be detected in HE 1327-2326. The upper limit ${ }^{9}$ is $\log \varepsilon(\mathrm{Li}) \leqslant 0.7$, in contrast to the Spite-plateau value of $\log \varepsilon(\mathrm{Li}) \approx 2.3$ found in other stars. This finding currently lacks a satisfying explanation.

In search for a solution, one can question the assumption that the surface abundances of old stars are constant over time. Even for single MS or SG stars, processes may be at work which systematically alter the chemical surface composition. For solar-type stars, stellar-structure models allowing the atoms to move freely inside the star under the prevailing forces predict sizeable effects in particular for old metal-poor stars. Such processes (gravitational settling and radiative levitation, to name two prominent ones) are collectively referred to as atomic diffusion and require billions of years to alter the abundances of solar-type stars. Convection can suppress their efficiency, but atomic diffusion seems capable of depleting the thin lowmass convective envelopes of metal-poor stars appreciably (see, e.g., Richard et al. 2002), unless extra mixing processes stir up the boundary layer between the convective and the radiative zone.

Observational evidence in favor of atomic diffusion in the presence of some extra mixing was recently presented (Korn et al. 2006, 2007; Lind et al. 2008) for stars in NGC 6397 $([\mathrm{Fe} / \mathrm{H}] \approx-2)$. Based on observed abundance trends between stars from the turnoff point (TOP) to the red-giant branch (RGB), the mixing efficiency needed in the stellar-structure models was empirically determined. This mixing falls in the range expected from observational constraints set by the Spite plateau (Richard et al. 2005); surface-abundance alterations due to atomic diffusion are then limited to $\pm 0.3 \mathrm{dex}$ and help to harmonize the inferred lithium abundances with the predictions from Wilkinson Microwave Anisotropy Probe (WMAP)-based big bang nucleosynthesis calculations (Korn et al. 2006).

In this paper, we thus explore to which extent the surface abundances of HE 1327-2326 may be affected by atomic diffusion. After describing the observational material (Section 2) we derive stellar parameters using spectrophotometry and NLTE diagnostics (Section 3). Based on these, we present upper limits on the abundance variations induced by atomic diffusion and discuss their consequences (Section 4). In Section 5, we summarize our conclusions.

\footnotetext{
9 The customary logarithmic 12 -scale is used here: $\log \varepsilon(\mathrm{X})=\log n_{\mathrm{X}} / n_{\mathrm{H}}+12$.
}

\section{OBSERVATIONS}

We combine data obtained in 2004 and 2005 with Subaru/ High Dispersion Spectrograph (HDS) and Very Large Telescope (VLT)/Ultraviolet and Visual Echelle Spectrograph (UVES), respectively. The UVES spectra $(R=40,000$; Frebel et al. 2006) are used for the Balmer lines $(\mathrm{H} \alpha$ and $\mathrm{H} \beta)$ and the Ca IR triplet lines. The HDS spectra $(R=60,000$; Aoki et al. 2006) cover Ca K $3933 \AA$ and the Ca I resonance line $4226 \AA$. In all cases, the signal-to-noise ratio exceeds $200: 1$. The limiting factor for an abundance analysis of HE 1327-2326 is thus not to be sought in the quality of the input data.

Spectrophotometric observations of HE 1327-2326 were obtained with the Double Beam Spectrograph (DBS) on the $2.3 \mathrm{~m}$ telescope at Siding Springs Observatory, Australia. In 2006 June, a "600B" spectrum from $3200 \AA$ to $5200 \AA$ was obtained. In 2009 January, a "300B" spectrum was taken covering the wavelength range from $3200 \AA$ to $6200 \AA$. The spectra were taken with the atmospheric dispersion along the slit to ensure that the relative absolute fluxes were accurately measured. The spectra were corrected for continuous atmospheric extinction and wavelength calibrated using $\mathrm{Ne}-\mathrm{Ar}$ arc exposures. They were also divided by a continuous white dwarf spectrum to remove the spectrograph and dichroic response as described by Bessell (1999, 2007). The spectra were then scrunched and flux corrected using more than five spectrophotometric standards selected each night from the Next Generation Stellar Library STIS observations of Michael Gregg ${ }^{10}$ as re-reduced by Heap and Lindler. ${ }^{11}$ The relative absolute fluxes of the NGSL stars are precise to within $2 \%$. The $300 \mathrm{~B}$ and $600 \mathrm{~B}$ spectra show excellent agreement.

\section{STELLAR PARAMETERS}

A crucial ingredient in this investigation is the knowledge of HE 1327-2326's evolutionary status. So far, it has not been possible to distinguish whether HE 1327-2326 is an MS or an SG star. Luckily, this has no significant impact on the abundances derived from minority species (like $\mathrm{Ca}$ I and $\mathrm{Fe}$ I) and only a small one for majority ones (like $\mathrm{Ca}$ II, $\approx 0.2$ $\mathrm{dex}$ ). For $\mathrm{C}, \mathrm{N}$, and $\mathrm{O}$, however, there are differences of up to $\approx 0.5$ dex between the two cases, as these abundances are exclusively derived from molecular features. Since the CNO abundances with respect to iron are extremely high in this star, these differences have not greatly hampered the interpretation of the overall abundance pattern. But with increasing sophistication of the modeling it is crucial to determine the evolutionary status of this star. This is also the case when comparing to predictions from stellar-structure models.

\subsection{Effective Temperature}

From four broadband indices calibrated on the infrared-flux method (IRFM) of Alonso et al. (1996), a mean effective temperature of $T_{\text {eff }}=6180 \mathrm{~K}$ was derived (Frebel et al. 2005; Aoki et al. 2006). Balmer lines in LTE seemed to favor a somewhat lower effective temperature, $T_{\text {eff }} \approx 6000 \mathrm{~K}$, irrespective of the broadening theory applied (see Table 7 of Aoki et al. 2006).

We have performed NLTE calculations for H I using the method described in Mashonkina et al. (2008). In brief, the

\footnotetext{
10 http://www.stsci.edu/hst/HST_overview/documents/calworkshop/workshop 2005/papers/gregg.pdf

11 http://archive.stsci.edu/prepds/stisngsl/index.html
} 


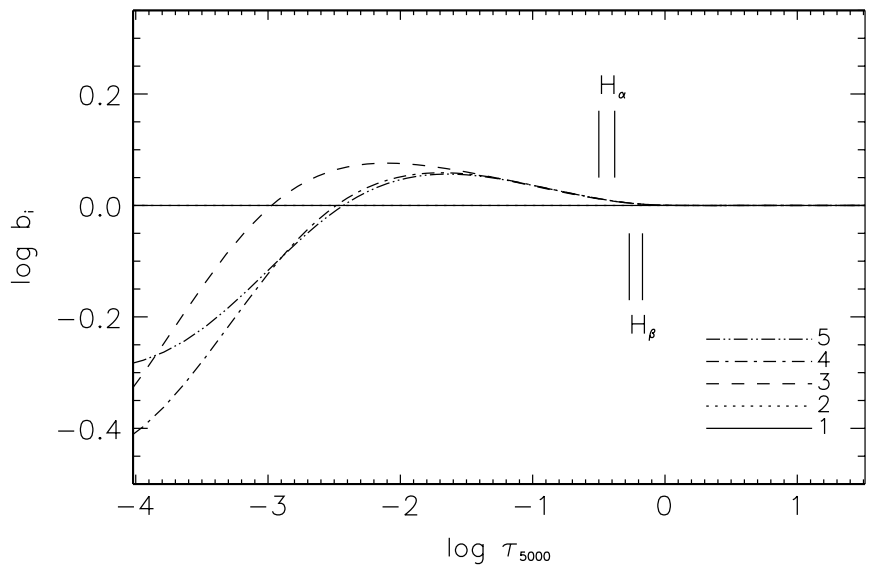

Figure 1. Departure coefficients $\log b_{i}$ for the five lowest levels of $\mathrm{H} \mathrm{I}$ in the model atmosphere 6180/3.7/-4.34. Tick marks indicate the locations of coreto-wing transition formation depths for $\mathrm{H}_{\alpha}$ and $\mathrm{H}_{\beta}$ (0.85-0.95 in normalized flux).

Table 1

Spectroscopic Effective Temperatures as Derived from Balmer Lines in NLTE

\begin{tabular}{lccc}
\hline \hline Star & $\mathrm{H} \alpha_{\text {NLTE }}$ & $\mathrm{H} \beta_{\text {NLTE }}$ & Error $^{\mathrm{a}}$ \\
\hline HE $1327-2326$ & $6120 \mathrm{~K}$ & $6120 \mathrm{~K}$ & $\pm 150 \mathrm{~K}$ \\
Sun & $5780 \mathrm{~K}$ & $5780 \mathrm{~K}$ & $+20 \mathrm{~K},-60 \mathrm{~K}$ \\
\hline
\end{tabular}

Note. ${ }^{\text {a }}$ Estimated total error budget including observational uncertainties.

model atom includes levels with principal quantum numbers up to $n=19$. Collisional rates include inelastic collisions with electrons and hydrogen atoms. For the latter, we use the formula of Steenbock \& Holweger (1984). Since it provides only an order of magnitude estimate, we apply it with a scaling factor $S_{\mathrm{H}}=$ 2 as favored by the consistency of the effective temperatures derived from two Balmer lines, $\mathrm{H} \alpha$ and $\mathrm{H} \beta$, in four metal-poor $([\mathrm{Fe} / \mathrm{H}]<-2)$ stars (Mashonkina et al. 2008).

For the model atmospheres investigated in this study, the ground state and the $n=2$ level keep their thermodynamicequilibrium level populations throughout the atmosphere. Figure 1 shows the behavior of the departure coefficients $b_{i}=n_{i}^{\mathrm{NLTE}} / n_{i}^{\mathrm{LTE}}$ of the relevant levels of $\mathrm{H} \mathrm{I}$ as a function of continuum optical depth $\tau_{5000}$ referring to $\lambda=5000 \AA$ in the

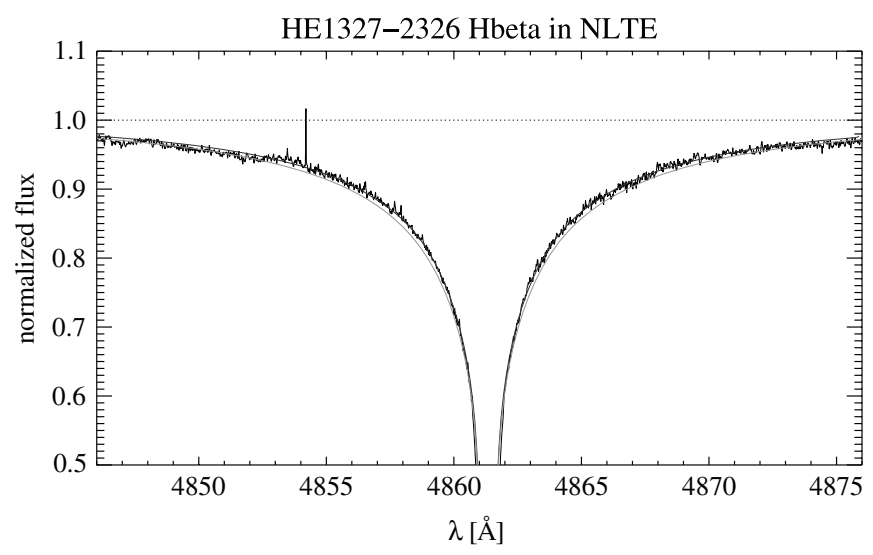

Figure 3. Fit to the observed $\mathrm{H} \beta$ profile (black line). An effective temperature of $T_{\text {eff }}=6120 \mathrm{~K}$ is indicated in NLTE. The predicted NLTE profile for $T_{\text {eff }}=$ $6180 \mathrm{~K}$ is indicated by the gray line.

model with $T_{\text {eff }} / \log g /[\mathrm{Fe} / \mathrm{H}]$ of $6180 / 3.7 /-4.34$. Here, $n_{i}^{\mathrm{NLTE}}$ and $n_{i}^{\mathrm{LTE}}$ are the statistical-equilibrium and thermal (SahaBoltzmann) number densities, respectively. Departures from LTE for the $n=3$ level are controlled by $\mathrm{H} \alpha$. In the layers where the continuum optical depth drops below unity $\mathrm{H} \alpha$ serves as the pumping transition resulting in an overpopulation of the upper level. For instance, $b(n=3) \approx 1.08$ around $\log \tau_{5000}=-0.5$ where the core-to-wing transition is formed. Starting from $\log \tau_{5000} \approx-3$ and further out, photon losses from the wings of $\mathrm{H} \alpha$ cause an underpopulation of the $n=3$ level. We obtain weak NLTE effects for the $\mathrm{H} \beta$ profile beyond the core (which is not considered in the analysis), independent of the applied scaling factor for hydrogen collisions. For $\mathrm{H} \alpha$, NLTE leads to a weakening of the core-to-wing transition compared to the LTE case. Exactly this part of profile is sensitive to $T_{\text {eff }}$ variations.

Table 1 summarizes our $T_{\text {eff }}$ determination. In NLTE, both $\mathrm{H} \alpha$ and $\mathrm{H} \beta$ indicate an effective temperature close to $T_{\text {eff }}=6120 \mathrm{~K}$ (see Figures 2 and 3 ) putting the new spectroscopic effective temperatures in much better agreement with the photometric estimates. For reasons of consistency with previous publications in this series, we adopt $T_{\text {eff }}=6180 \mathrm{~K}$ as our current best estimate (a significantly higher effective temperature seems possible using different calibrations of the IRFM (Ramírez \& Meléndez 2005 ) and we briefly discuss the impact of this scenario below).

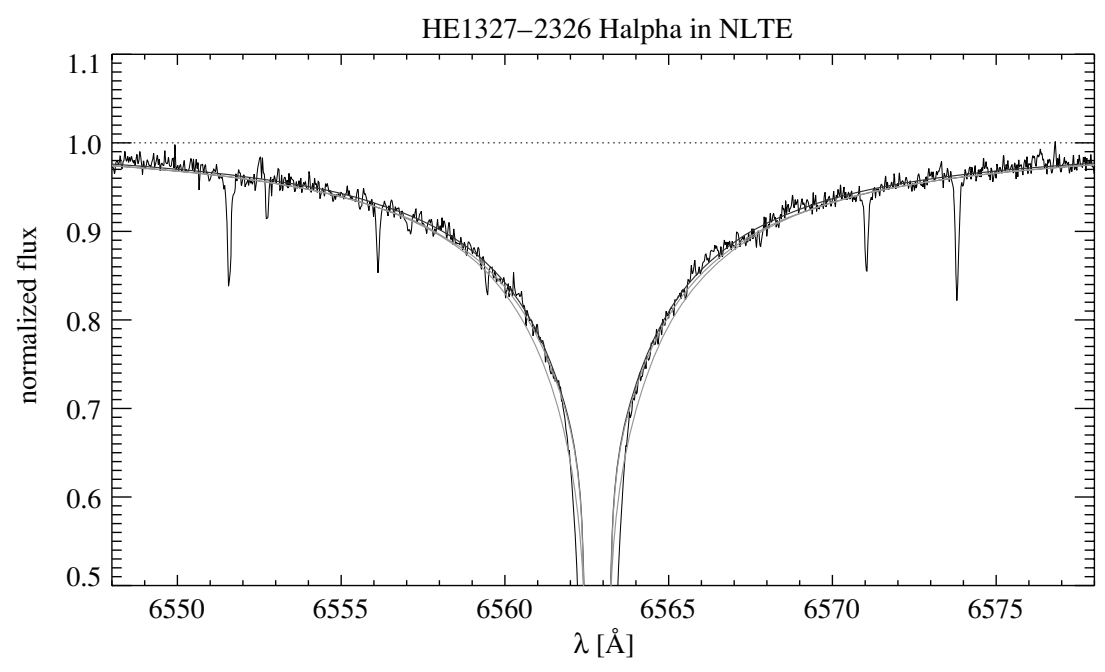

Figure 2. Fit to the observed $\mathrm{H} \alpha$ profile. An effective temperature of $T_{\text {eff }}=6120 \mathrm{~K}$ is indicated in NLTE. The predicted NLTE profile for $T_{\text {eff }}=6180 \mathrm{~K}$ is indicated by the upper gray line, the LTE one by the lower one. 


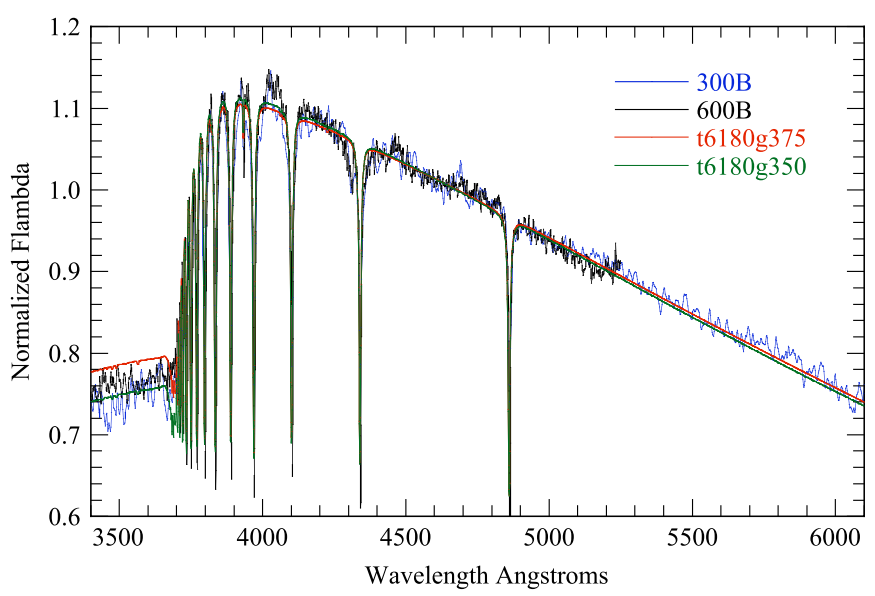

Figure 4. Comparison between spectrophotometric observations of $\mathrm{HE}$ $1327-2326$ and model-atmosphere predictions $\left(T_{\mathrm{eff}}=6180 \mathrm{~K},[\mathrm{Fe} / \mathrm{H}]=-5\right.$ $E(B-V)=0.075)$. The strength of the Balmer jump indicates a surface gravity of $\log g=3.65$.

(A color version of this figure is available in the online journal.)

\subsection{Surface Gravity}

The surface gravity of HMP stars is, generally speaking, difficult to constrain observationally. The Balmer jump can serve as a photometric surface-gravity indicator (see, e.g., Önehag et al. 2008). Figure 4 shows comparisons between the two DBS observations and model fluxes of two MARCS models (Gustafsson et al. 2008) with $T_{\text {eff }}=6180 \mathrm{~K},[\mathrm{Fe} / \mathrm{H}]=-5$ and $\log g$ between 3.5 and $3.75(E(B-V)=0.075$ was assumed). As the recent "300B" spectrum was obtained in bright time, higher weight is given to the " $600 \mathrm{~B}$ " observations. A spectrophotometric surface gravity of close to $\log g=3.65$ is derived.

Ionization equilibria are a standard spectroscopic method of constraining $\log g$. The typical attainable precision is $0.3 \mathrm{dex}$ when line-to-line scatter is of order 0.1 dex. The accuracy can be increased by considering NLTE effects. The present investigation of Ca I/II in NLTE is based on our earlier work (Mashonkina et al. 2007) where atomic data and the problems of calcium line formation were considered in detail. The model atom used is up-to-date and includes effective collision strengths for $\mathrm{Ca}$ II transitions from recent $R$-matrix calculations of Meléndez et al. (2007). Inelastic collisions with neutral $\mathrm{H}$ particles are accounted for using the formula of Steenbock \& Holweger (1984) with a scaling factor $S_{\mathrm{H}}=0.1$ as empirically determined from their different influence on $\mathrm{Ca}$ I and $\mathrm{Ca}$ II lines in solar and stellar spectra. As in the case of $\mathrm{H}$, such a scaling is warranted, as the Steenbock \& Holweger (1984) recipe yields collisional rates with large systematic uncertainties.

NLTE calculations are performed for two MAFAGS (Fuhrmann et al. 1997) model atmospheres with effective temperature $T_{\text {eff }}=6180 \mathrm{~K}$ and iron abundance $[\mathrm{Fe} / \mathrm{H}]=-4.34$, but with different surface gravities, $\log g=3.7$ and 4.5 (the input calcium abundance is iteratively adjusted to match the derived value). The behavior of the departure coefficients and the line source functions is similar for both models. In the first place, we are interested in the behavior of the lower and upper levels from which the investigated lines, Ca I $4226 \AA$, Ca II $3933 \AA$, and Ca II $8498 \AA$, arise. Figure 5 shows the departure coefficients of the selected levels as a function of continuum optical depth $\tau_{5000}$ in the model $6180 / 3.7 /-4.34$.

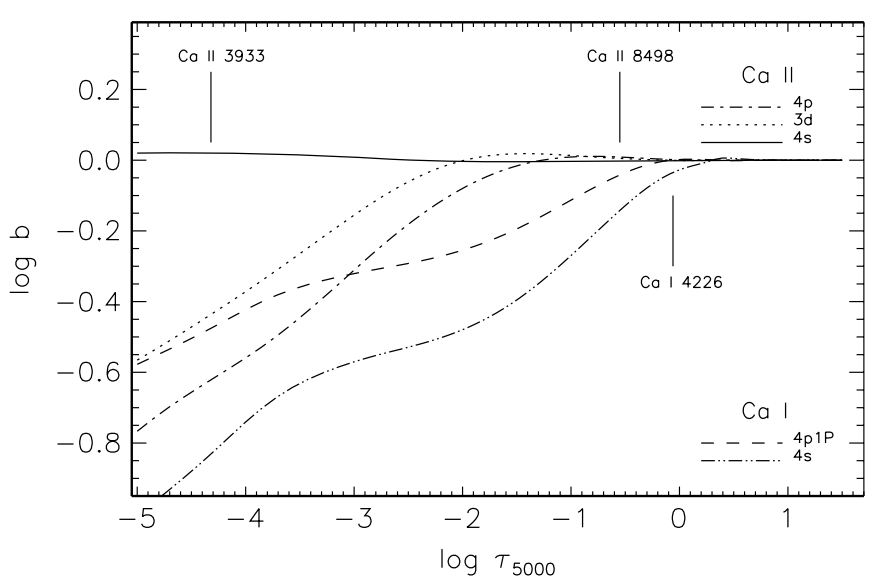

Figure 5. Departure coefficients $\log b_{i}$ for selected levels of $\mathrm{Ca}$ I and Ca II in the model atmosphere 6180/3.7/-4.34. Tick marks indicate the locations of line-center optical depth unity for Ca I $4226 \AA$, Ca II $3933 \AA$ A, and Ca II $8498 \AA$.

For the minority species Ca I, the main NLTE effect is overionization. It is caused by superthermal radiation of nonlocal origin below the thresholds of the Ca I ground state $4 s^{2}{ }^{1} \mathrm{~S}\left(\lambda_{\text {thr }}\right.$ $=2028 \AA)$ and the low-excitation levels $4 p^{3} P^{\circ}\left(\lambda_{\text {thr }}=2930 \AA\right)$ and $3 d^{3} \mathrm{D}\left(\lambda_{\text {thr }}=3450 \AA\right)$. The Ca I resonance line is weaker relative to its LTE strength not only due to the general overionization but also due to the larger departure coefficient of the upper level compared to that for the lower one resulting in a line source function $S_{l u} \simeq b\left(4 p^{1} P^{\circ}\right) / b\left(4 s^{2}{ }^{1} \mathrm{~S}\right) B_{v}>B_{v}$ and diminished line absorption. The NLTE abundance correction $\Delta_{\mathrm{NLTE}}=\log \varepsilon(\mathrm{NLTE})-\log \varepsilon(\mathrm{LTE})=+0.31 \mathrm{dex}$ and $+0.25 \mathrm{dex}$ for the models $6180 / 3.7 /-4.34$ and $6180 / 4.5 /-4.34$, respectively.

In the line formation layers, $\mathrm{Ca}$ II dominates the element number density. No process seems to affect the Ca II groundstate population and $4 s$ keeps its thermodynamic-equilibrium value. Ca II resonance-line pumping produces a slightly enhanced excitation of the level $4 p$ in the atmospheric layers between $\log \tau_{5000}=-0.3$ and -1.3 . In the very metal-poor atmosphere of HE 1327-2326, the metastable level $3 d$ is only weakly coupled to the ground state and follows $4 p$ until photon losses in the Ca II 8498 line start to become important. Departures from LTE for Ca II 8498 are weak resulting in $\Delta_{\text {NLTE }}$ $=-0.02 \mathrm{dex}$ and $-0.01 \mathrm{dex}$ for the models $6180 / 3.7 /-4.34$ and $6180 / 4.5 /-4.34$, respectively. Because of the larger line strength (and thus shallower formation depth) of Ca II 3933, this line suffers from somewhat larger NLTE effects: $-0.15 \mathrm{dex}$ and -0.06 dex for the subgiant and dwarf models, respectively.

We note that NLTE effects for Ca I/II in 3D line formation calculations are currently unexplored. For the minority species Li I in metal-poor stellar atmospheres, Barklem et al. (2003) concluded that $3 \mathrm{D}+$ NLTE is not greatly different from $1 \mathrm{D}+$ NLTE with one-dimensional + NLTE results overestimating the Li abundance by up to 0.1 dex. Similar effects can be expected for Ca I. For Ca II, the departures from LTE are likely to be small, similar to the $1 \mathrm{D}$ case.

Table 2 summarizes the results concerning calcium. Good agreement (within $0.05 \mathrm{dex}$ ) is obtained for the three different lines when NLTE and a surface gravity of $\log g=3.7$ are used (numerically, a slightly lower $\log g$ value is indicated which we disregard for reasons explained in Section 4). At the dwarf-like surface gravity, the $\log g$-sensitive line Ca II 8498 is more than 0.4 dex discrepant from $\mathrm{Ca}$ I 4226 . From this and the line-to-line scatter we derive an uncertainty for $\log g$ of 0.2 dex. 
Table 2

Calcium Abundances as Derived from Individual Lines

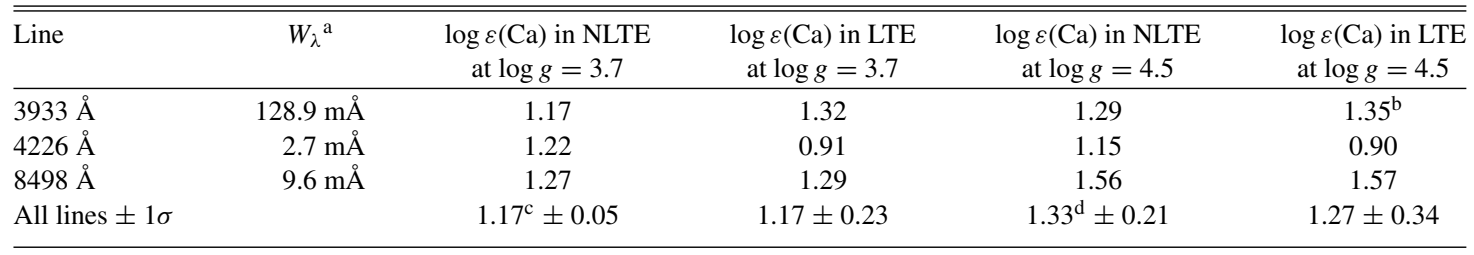

Notes. Like in Aoki et al. (2006), a microturbulence value of $1.7 \mathrm{~km} \mathrm{~s}^{-1}$ and $1.5 \mathrm{~km} \mathrm{~s}^{-1}$ was assumed in the line formation calculations for the subgiant and dwarf, respectively.

a As determined from the theoretical spectra after profile fitting.

${ }^{b}$ In this analysis, the fit is based on the wings, as the wings and core cannot be fitted simultaneously.

${ }^{\mathrm{c}}$ Corresponds to $[\mathrm{Ca} / \mathrm{Fe}]=0.47$ on the scale of Aoki et al. (2006).

${ }^{\mathrm{d}}$ Corresponds to $[\mathrm{Ca} / \mathrm{Fe}]=0.62$ on the scale of Aoki et al. (2006).

Table 3

Initial Chemical Composition of the Two Different Diffusion Models

\begin{tabular}{|c|c|c|}
\hline \multirow[t]{2}{*}{ Element } & \multicolumn{2}{|c|}{ Mass Fraction } \\
\hline & {$[\mathrm{Fe} / \mathrm{H}]=-5.61, \mathrm{~T} 6.0$} & {$[\mathrm{Fe} / \mathrm{H}]=-7.11$, Diffusive } \\
\hline $\mathrm{H}$ & $7.642 \times 10^{-1}$ & $7.645 \times 10^{-1}$ \\
\hline${ }^{4} \mathrm{He}$ & $2.354 \times 10^{-1}$ & $2.354 \times 10^{-1}$ \\
\hline${ }^{7} \mathrm{Li}$ & $2.0 \times 10^{-9}$ & $2.0 \times 10^{-9}$ \\
\hline${ }^{12} \mathrm{C}^{\mathrm{a}}$ & $8.633 \times 10^{-5}$ & $2.763 \times 10^{-6}$ \\
\hline $\mathrm{N}^{\mathrm{a}}$ & $2.647 \times 10^{-5}$ & $8.471 \times 10^{-7}$ \\
\hline $\mathrm{O}^{\mathrm{a}}$ & $2.409 \times 10^{-4}$ & $7.708 \times 10^{-6}$ \\
\hline $\mathrm{Ne}$ & $4.927 \times 10^{-9}$ & $1.577 \times 10^{-10}$ \\
\hline $\mathrm{Na}$ & $9.989 \times 10^{-11}$ & $3.197 \times 10^{-12}$ \\
\hline $\mathrm{Mg}$ & $1.875 \times 10^{-9}$ & $6.002 \times 10^{-11}$ \\
\hline $\mathrm{Al}$ & $1.623 \times 10^{-10}$ & $5.194 \times 10^{-12}$ \\
\hline $\mathrm{Si}$ & $2.023 \times 10^{-9}$ & $6.473 \times 10^{-11}$ \\
\hline $\mathrm{P}$ & $1.748 \times 10^{-11}$ & $5.594 \times 10^{-13}$ \\
\hline S & $1.056 \times 10^{-9}$ & $3.380 \times 10^{-11}$ \\
\hline $\mathrm{Cl}$ & $2.248 \times 10^{-11}$ & $7.192 \times 10^{-13}$ \\
\hline $\mathrm{Ar}$ & $2.697 \times 10^{-10}$ & $8.631 \times 10^{-12}$ \\
\hline $\mathrm{K}$ & $9.989 \times 10^{-12}$ & $3.197 \times 10^{-13}$ \\
\hline $\mathrm{Ca}$ & $1.873 \times 10^{-10}$ & $5.994 \times 10^{-12}$ \\
\hline $\mathrm{Ti}$ & $9.989 \times 10^{-12}$ & $3.197 \times 10^{-13}$ \\
\hline $\mathrm{Cr}$ & $4.994 \times 10^{-11}$ & $1.598 \times 10^{-12}$ \\
\hline $\mathrm{Mn}$ & $2.747 \times 10^{-11}$ & $8.791 \times 10^{-13}$ \\
\hline $\mathrm{Fe}$ & $3.586 \times 10^{-9}$ & $1.147 \times 10^{-10}$ \\
\hline $\mathrm{Ni}$ & $2.223 \times 10^{-10}$ & $7.112 \times 10^{-12}$ \\
\hline $\mathrm{Z}$ & $3.545 \times 10^{-4}$ & $1.135 \times 10^{-5}$ \\
\hline
\end{tabular}

Notes. The abundance variations after $\approx 13.3 \mathrm{Gyr}$ can be estimated from Figure 7.

${ }^{\text {a }}$ Increased by +4 dex relative to the assumed solar mixture.

A rigorous NLTE modeling of $\mathrm{Ca} \mathrm{I} / \mathrm{II}$ in 1D is thus capable of fully accounting for the $\mathrm{Ca}$ I/II abundance discrepancy seen in previous analyses. HE $1327-2326$ is therefore a subgiant, a conclusion in accord with indications from Balmer lines (see Aoki et al. 2006). The $[\mathrm{Ca} / \mathrm{Fe}]$ ratio is $+0.47 \mathrm{dex}$ which is not an unusual value for a halo star. HE 1327-2326 is a benchmark case for future NLTE studies for $\mathrm{Ca}$ I/II in 3D model atmospheres.

We estimate the effects of crucial atomic data on the accuracy of the NLTE Ca abundances derived from Ca I 4226 and $\mathrm{Ca}$ II 8498 (the two most critical lines for our purpose) using the model 6180/3.7/-4.34. The largest effects are found upon disregarding hydrogenic collisions altogether. But even in that case, none of the line abundances changes by more than $0.1 \mathrm{dex}$ and our conclusion about HE 1327-2326 being a subgiant holds.

\section{STELLAR-STRUCTURE MODEL PREDICTIONS}

\subsection{Model Description}

The stellar evolutionary models used here are calculated with the physics of particle transport that is known from first principles. In radiative zones this implies taking into account atomic diffusion including gravitational settling, thermal diffusion, and radiative accelerations, in addition to the purely diffusive term. The diffusion velocity of each species is computed with the equations as developed in Burgers (1969) which take into account the interactions between all diffusing species.

The detailed treatment of atomic diffusion is described in Turcotte et al. (1998). In these models, the Rosseland opacity and radiative accelerations are computed in each layer for the exact local chemical composition using OPAL monochromatic opacities and for each time step during the evolution. The radiative accelerations are from Richer et al. (1998) with corrections for redistribution from Gonzalez et al. (1995) and LeBlanc et al. (2000). Convection and semi-convection are modeled as diffusion processes as described in Richer et al. (2000) and Richard et al. (2001).

In one set of models, additional mixing is included in the diffusion equation by adding an effective diffusion coefficient as in Richer et al. (2000) and Richard et al. (2001). The parameters specifying this effective diffusion coefficients are indicated in the name assigned to the model, which are the same as those used in the Korn et al. (2007) study on NGC 6397. This additional mixing is introduced in an ad hoc manner: here no attempt is made to connect it to physical processes like rotation. As shown by Richer et al. (2000), the most important factor is the radial extent of the mixed layers. All models considered here were assumed to be chemically homogeneous on the pre-main sequence with solar relative mass fractions as given in Table 3 . To mimic the abundance peculiarities of HE 1327-2326, the relative concentrations of the $\mathrm{CNO}$ elements were increased by +4 dex compared with the scaled solar mixture. As the most abundant metals in HE 1327-2326, they have structural effects on the models.

\subsection{Best-Fitting Models}

We computed a variety of models with masses around $0.78 M_{\odot}$ for this study, both purely diffusive ones and those including additional mixing. The mass and initial composition of each model was varied to roughly meet the observational constraints of HE 1327-2326: the surface abundance of iron should be within a factor of 2 of $[\mathrm{Fe} / \mathrm{H}]=-5.8$ (the average of the 1D and 3D LTE abundances derived by Aoki et al. 2006 


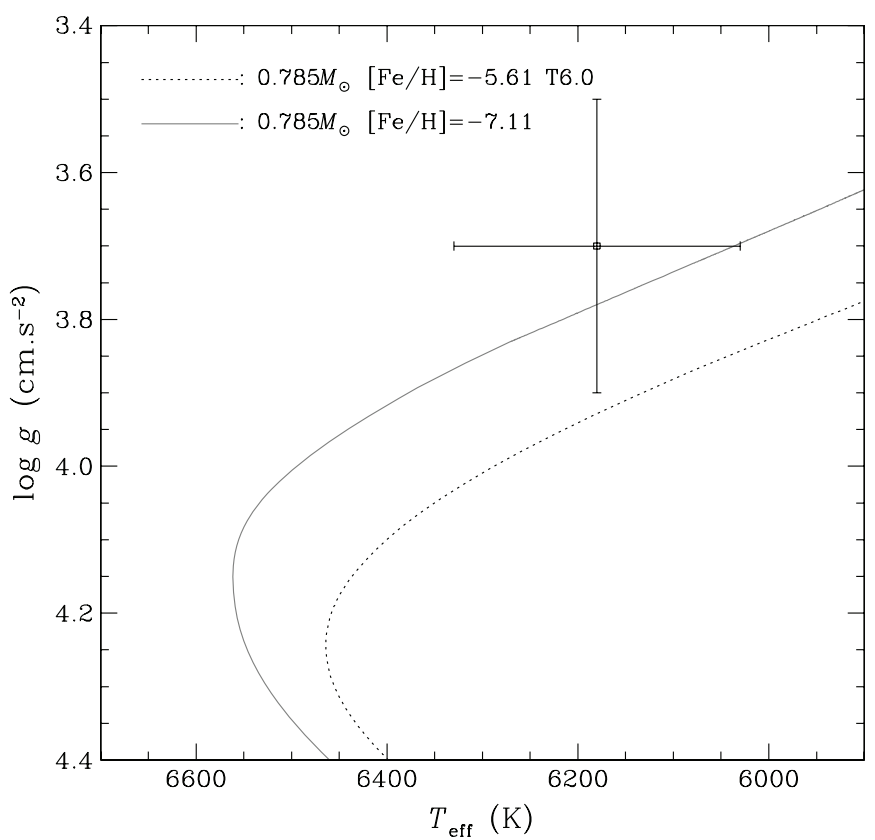

Figure 6. Position of HE 1327-2326 relative to evolutionary models constructed from the two diffusion models.

and Frebel et al. 2008a) when the model star reaches the error ellipse of HE 1327-2326 on the subgiant branch after roughly 13 Gyr (see Figure 6).

Two models of $0.785 M_{\odot}$ fulfilled these a posteriori requirements. The first one has an initial $[\mathrm{Fe} / \mathrm{H}]$ of $[\mathrm{Fe} / \mathrm{H}]_{i}=-7.11$ and was computed with atomic diffusion and radiative accelerations, but without additional transport processes in the radiative zone. As shown by Richard et al. (2002), for higher metallicities (between -2.31 and -4.31 ), such a star is expected to display strong surface abundance variations close to and past the TOP. The second one $([\mathrm{Fe} / \mathrm{H}]=-5.61)$ additionally includes additional transport as needed to explain abundance variations of NGC 6397 (Korn et al. 2006, 2007).

\subsection{Birth-Cloud versus Present-Day Abundances}

As can be seen from Figure 7, abundance corrections are large for the model with uninhibited diffusion: after $\approx 13.4 \mathrm{Gyr}$ the $M=0.785 M_{\odot}[\mathrm{Fe} / \mathrm{H}]_{i}=-7.11$ model has reached the subgiant branch and its surface lithium is depleted by almost 1.2 dex. Simultaneously, radiative levitation has increased its convection-zone abundances of iron by 1.2 dex. Other elements, e.g., aluminum and nickel, are affected by an even larger amount. We compared the diffusion-corrected abundance pattern of HE 1327-2326 to that of HE 0107-5240 and find that the abundance patterns of the two stars do not become more similar. When additional mixing is introduced into the model, the surface-abundance changes are largely moderated: helium and lithium only settle by $\approx 0.3$ dex (incidentally, the same amount as predicted for NGC 6397; Korn et al. 2007), iron by only $\approx 0.1 \mathrm{dex}$.

If HE 1327-2326 turned out to be significantly hotter $\left(T_{\text {eff }}=\right.$ $6450 \mathrm{~K}$ ), then a surface gravity of $\log g \approx 4.0$ would be derived in the framework of this analysis. From the stellar-structure point of view, this is a meaningful combination of stellar parameters (see Figure 6), albeit not supported by our understanding of the formation of Balmer lines. The same diffusion models could be used to predict corrections to the surface abundances and these would turn out to be quite similar within a given set of

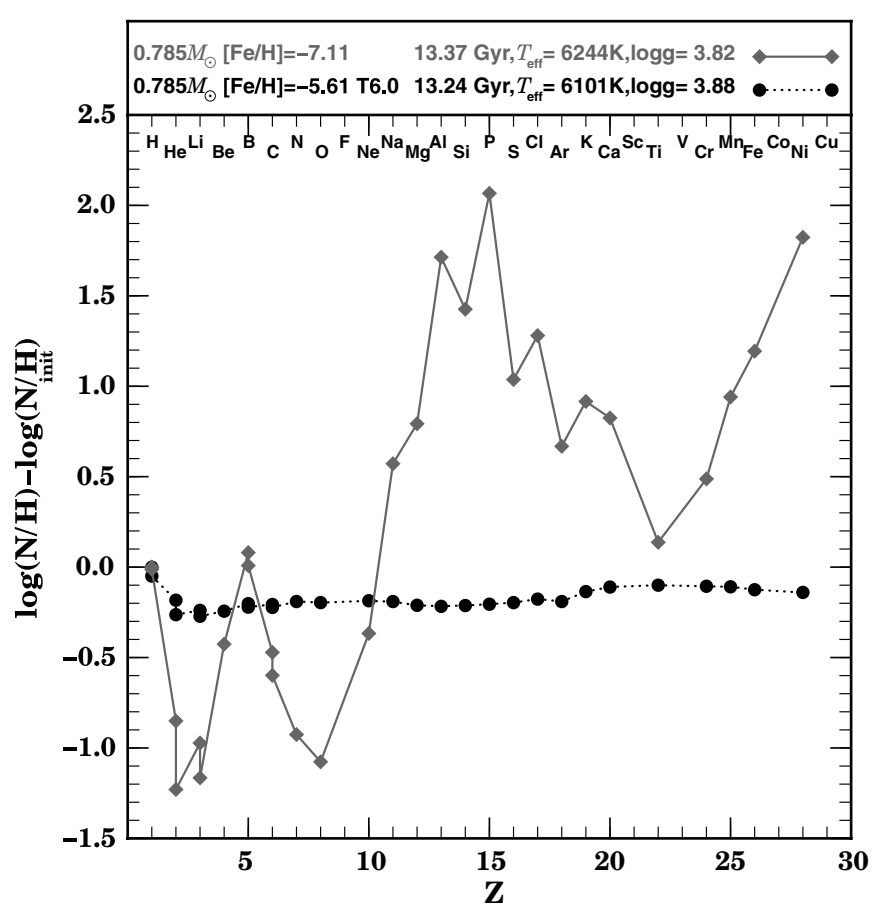

Figure 7. Surface-abundance variations as predicted by the two diffusion models, relative to the initial abundances given in Table 3 .

model assumptions. This is owed to the fact that the change in stellar parameters effectively moves the star along its fast early post-TOP evolutionary track.

Given our present ignorance of the extent of the additional mixing (and how it scales with, e.g., metallicity), it is difficult to draw firm conclusions about which of the two diffusion scenarios is more realistic. It is conceivable that the mixing efficiency declines toward lower metallicities, as the convective envelope decreases in extent and mass. Nonetheless, even in the absence of mixing the correction is not large enough to reconcile the upper limit on the lithium abundance $(\log \varepsilon(\mathrm{Li}) \leqslant 0.7)$ with the WMAP-based primordial lithium-abundance prediction of big bang nucleosynthesis $(\log \varepsilon(\mathrm{Li})=2.63$; Spergel et al. 2007). If the low lithium content of HE 1327-2326 turned out to be the rule among unevolved HMP star, then this may tell us something specific about the environment these stars were able to form in. More work is needed to identify similar stars in the outer halo.

Until better constraints can be set on the mixing efficiency, we recommend to use the abundance corrections based on the model with extra mixing. These corrections are moderate and generally negative. The birth cloud from which HE 1327-2326 formed was somewhat more metal-rich than the surface abundances of HE 1327-2326 show at the present time.

As discussed in Korn et al. (2007), helium diffusion has a structural effect on the atmosphere of a star and can be mapped as a shift in the surface-gravity scale. The gravitational settling of helium entails a shift of the surface gravity of $\approx+0.05$ dex. This correction was considered when deriving our best current estimate of $\log g=3.7 \pm 0.2$.

\section{CONCLUSIONS}

We have derived spectroscopic stellar parameters for $\mathrm{HE}$ 1327-2326 using 1D NLTE techniques: Balmer line profiles in NLTE yield $T_{\text {eff }}=6120 \pm 150 \mathrm{~K}$ confirming the photometrically determined effective temperature of $T_{\text {eff }}=6180 \mathrm{~K}$. The calcium 
ionization equilibrium in NLTE points toward a subgiant surface gravity of $\log g=3.7 \pm 0.2$, as does spectrophotometry of the Balmer jump. Using an effective temperature of $6450 \mathrm{~K}$ (as favored by a revision of the IRFM; Ramírez \& Meléndez 2005), results in a surface gravity typical for turnoff stars. While this is also a sensible stellar-parameter combination from the point of view of stellar evolution and the $\mathrm{Ca}$ I/II ionization equilibrium, it finds no support from Balmer-line analyses.

The exploratory investigations into how much $\mathrm{HE}$ 1327-2326's surface abundances may be affected by atomic diffusion show that large (more than 1 dex) corrections are in principle possible. The material from which HE 1327-2326 formed would then have been more metal-poor $\left([\mathrm{Fe} / \mathrm{H}]_{i} \approx-7\right.$ ) and significantly more lithium-rich $\left(\log \varepsilon(\mathrm{Li})_{i} \leqslant 1.9\right)$. However, there is no compelling evidence in favor of uninhibited diffusion in stars that have convective envelopes. If additional mixing below the convective envelope is postulated (as needed to explain abundance trends in NGC 6397; Korn et al. 2007), then the abundance corrections are limited to a factor of 2 . Assuming the additional-mixing efficiency to be independent of metallicity, the corrections are thus moderate given the uncertainties associated with present-day 1D (N)LTE or 3D LTE abundances of HE 1327-2326 and other HMP stars.

It is too early to draw firm conclusions about our ability to reliably read the chemical fingerprints in the atmospheres of stars like HE 1327-2326 and their nucleosynthetic significance as such. More such stars need to be identified in deep wide-angle surveys to leave the realm of low-number statistics. Efforts in this direction are under way. Systematic comparisons between chemical abundances in HMP dwarfs, subgiants, and giants may then reveal the efficiency of mixing moderating atomic diffusion. Simultaneously, the modeling has to be improved. This will mean replacing the hydrostatic approximation with full radiation hydrodynamics, both in stellar-structure and stellaratmosphere modeling. A similar transition is required for photospheric line-formation calculations in terms of statistical equilibria rather than LTE, as documented by the successes of this NLTE study.

A.J.K. acknowledges a research fellowship by the Swedish Research Council (Vetenskaprådet). O.R. thanks the Centre Informatique National de l'Enseignement Supérieur (CINES) and the Réseau Québécois de Calcul de Haute Performance (RQCHP) for providing the computational resources required for this work. M.L. is supported by Presidium RAS Programme "Origin and evolution of stars and the Galaxy" and the Deutsche Forschungsgemeinschaft with grant GE 490/34-1. A.F. is sup- ported through the W. J. McDonald Fellowship of the McDonald Observatory. W.A. is supported by a Grant-in-Aid for Science Research from JSPS (grant 18104003).

\section{REFERENCES}

Alonso, A., Arribas, S., \& Martinez-Roger, C. 1996, A\&A, 313, 873

Aoki, W., et al. 2006, ApJ, 639, 897

Barklem, P. S., Belyaev, A. K., \& Asplund, M. 2003, A\&A, 409, L1

Bessell, M. S. 1999, PASP, 111, 1426

Bessell, M. S. 2007, PASP, 119, 605

Burgers, J. M. 1969, Flow Equations for Composite Gases (New York: Academic Press)

Charbonnel, C., \& Primas, F. 2005, A\&A, 442, 961

Christlieb, N., et al. 2002, Nature, 419, 904

Frebel, A., Christlieb, N., Norris, J. E., Aoki, W., \& Asplund, M. 2006, ApJ, 638, L17

Frebel, A., Collet, R., Eriksson, K., Christlieb, N., \& Aoki, W. 2008a, ApJ, 684, 588

Frebel, A., Johnson, J. L., \& Bromm, V. 2008b, MNRAS, 392, L50

Frebel, A., et al. 2005, Nature, 434, 871

Fuhrmann, K., Pfeiffer, M., Frank, C., Reetz, J., \& Gehren, T. 1997, A\&A, 323, 909

Gonzalez, J.-F., LeBlanc, F., Artru, M.-C., \& Michaud, G. 1995, A\&A, 297 223

Gustafsson, B., Edvardsson, B., Eriksson, K., Jørgensen, U. G., Nordlund, А̊., \& Plez, B. 2008, A\&A, 486, 951

Iwamoto, N., Umeda, H., Tominaga, N., Nomoto, K., \& Maeda, K. 2005, Science, 309, 451

Korn, A. J., Grundahl, F., Richard, O., Barklem, P. S., Mashonkina, L., Collet, R., Piskunov, N., \& Gustafsson, B. 2006, Nature, 442, 657

Korn, A. J., Grundahl, F., Richard, O., Mashonkina, L., Barklem, P. S., Collet, R., Gustafsson, B., \& Piskunov, N. 2007, ApJ, 671, 402

LeBlanc, F., Michaud, G., \& Richer, J. 2000, ApJ, 538, 876

Lind, K., Korn, A. J., Barklem, P. S., \& Grundahl, F. 2008, A\&A, 490, 777

Mashonkina, L., Korn, A. J., \& Przybilla, N. 2007, A\&A, 461, 261

Mashonkina, L., et al. 2008, A\&A, 478, 529

Meléndez, M., Bautista, M. A., \& Badnell, N. R. 2007, A\&A, 469, 1203

Meynet, G., Ekström, S., \& Maeder, A. 2006, A\&A, 447, 623

Norris, J. E., Christlieb, N., Korn, A. J., Eriksson, K., Bessell, M. S., Beers, T. C., Wisotzki, L., \& Reimers, D. 2007, ApJ, 670, 774

Önehag, A., Gustafsson, B., Eriksson, K., \& Edvardsson, B. 2008 arXiv:0812.0388

Ramírez, I., \& Meléndez, J. 2005, ApJ, 626, 465

Richard, O., Michaud, G., \& Richer, J. 2001, ApJ, 558, 377

Richard, O., Michaud, G., \& Richer, J. 2002, ApJ, 580, 1100

Richard, O., Michaud, G., \& Richer, J. 2005, ApJ, 619, 538

Richer, J., Michaud, G., Rogers, F., Iglesias, C., Turcotte, S., \& LeBlanc, F. 1998, ApJ, 492, 833

Richer, J., Michaud, G., \& Turcotte, S. 2000, ApJ, 529, 338

Spergel, D. N., et al. 2007, ApJS, 170, 377

Spite, M., \& Spite, F. 1982, Nature, 297, 483

Steenbock, W., \& Holweger, H. 1984, A\&A, 130, 319

Turcotte, S., Richer, J., Michaud, G., Iglesias, C. A., \& Rogers, F. J. 1998, ApJ, 504,539

Venn, K. A., \& Lambert, D. L. 2008, ApJ, 677, 572 